\title{
On the pre-inflationary dark sector fields influence on the cosmological perturbations
}

\section{Olga Panina*}

Samara State Aerospace University

E-mail: panina.ph@gmail.com

\section{Sergey Chervon}

Ulyanovsk State Pedagogical University

E-mail: Chervon.sergeydgmail.com

\begin{abstract}
We suppose the appearance of the dark sector fields in the universe (eg., the quintessence, the phantom, and the axion field) during the pre-inflationary stage of the evolution. Moreover, these fields are describing by the nonlinear sigma model (NSM) and are considered weak, that is, the dynamical equations of this fields are considered on the inflationary background. The energy characteristics of the dark sector fields presumably of the same order as the perturbations of the gravitational field and the inflaton. We investigated the effects of the dark sector fields influence on the large-scale structure formation on the example of exponential inflation. We found that the dark sector fields reduce the perturbation of the gravitational field in this model.
\end{abstract}

The XXth International Workshop High Energy Physics and Quantum Field Theory September 24-October 1, 2011

Sochi Russia

\footnotetext{
* Speaker.
} 
Introduction. To understand the origin of dark energy as a source of modern accelerated expansion of the Universe, which was found from observations of the supernovae Ia, in the last decade have been developed theoretical models, including based on the cosmological constant $\Lambda$ and it's dynamical version [四]. In this study among all classes dark energy models, the models with scalar fields and the models with interacting fields are the most interesting. Others models

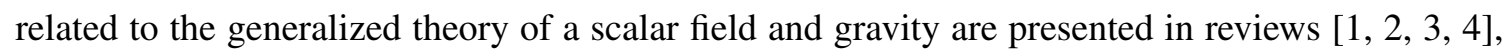
and in [వ] proposed a classification of such models, which includes 11 directions. Our approach is associated with scalar fields and interacting fields, which correspond to following directions (by the classification in [[]]): ii) models of the quintessence like gravitating self-interacting scalar field; iv) phantom fields; vi) interacting dark matter and dark energy fields.

We propose, that the dark sector fields was formed in pre-inflationary stage and exist in the inflationary stage as a weak fields. As a model which consists of the dark sector fields, we consider chiral cosmological model [ []]. In this case chiral fields $\varphi^{C}$, represents by dark sector fields, which we assume to be weak during inflation, and effects on the level of the inflaton perturbations $\phi$ : $\delta \phi \sim \varphi^{C}$ are only possible. In this model, dark sector field do not influence on the dynamics of the expansion process, but can correct the resulting large-scale structure. In this paper we investigate the effect on cosmological perturbations of the inflaton, which produced by two dark sector fields, that one of them may be a phantom field.

The method of calculating cosmological perturbations in the presence of a weak field (which is not the source of the gravitational field) in the background of Friedmann cosmology considered in [6], where they used the term «stiff source» instead of «weak field». As an example of stiff sources in cosmology may cite a cosmic strings, domain walls and textures. An investigation nonlinear sigma model as a «stiff» source realize in [四].

Basic equations of the model. We assume that the universe filled with a scalar field (inflaton) during the inflationary stage of evolution, which is the source of the gravitational field. On this self-gravitating system background we are considered a dark sector fields with the potential and kinetic interaction, which is described by the nonlinear sigma model with potential. Thus in this formulation of the problem at first we will solve the equations of the chiral fields dynamics on the background of the exact solution of equations of inflationary evolution of the universe [ $[8]$. Then proceed to consider the perturbed equations taking into account the stress-energy tensor of the weak fields. To describe the Universe during the inflationary stage by the cited above way, consider the action integral

$$
S=\int \sqrt{-g} d^{4} x\left[\frac{R}{2 \kappa}+\frac{1}{2} \phi_{, \mu} \phi_{, v} g^{\mu v}-V(\phi)+\frac{1}{2} h_{A B}\left(\varphi^{C}\right) \varphi_{, \mu}^{A} \varphi_{, v}^{B} g^{\mu v}-W\left(\varphi^{C}\right)\right]
$$

and impose the necessary constraints on the kinetic and potential components of the model. For simplicity we proceed to a two-component NSM with a targets space metric in the Gaussian coordinate system $d \sigma^{2}=d \psi^{2}+h_{22}(\psi, \chi) d \chi^{2}$. Let us represent the potential of interaction in the form of two terms $W^{(0)}(\phi, \psi, \chi)=V(\phi)+W(\psi, \chi)$, at the same time, think that $W(\psi, \chi) \ll V(\phi)$, so $W^{(0)} \approx V(\phi)$. We introduce a similar relation for the kinetic terms [ [Q] ]. We have $K(\phi, \psi, \chi)=$ $K^{(0)}(\phi)+K_{12}(\psi, \chi)$, where $K^{(0)}(\phi)=\frac{1}{2} \phi_{, \alpha} \phi^{, \alpha}, K_{12}(\psi, \chi)=\frac{1}{2} \psi_{, \alpha} \psi^{, \alpha}+\frac{1}{2} h_{22} \chi, \alpha \chi^{, \alpha}$. Assuming $K_{12} \ll K^{(0)}$ we get $K \approx K^{(0)}$. This restrictions can not take into account the chiral field $\psi, \chi$ in the Einstein equations. 
Thus, in consideration of this model with action $(\mathbb{W})$ and allowance for introduced approximations, we can use the exact solutions of the self-gravitating inflaton field equations, some examples you can find in the work [ए]]. Note that in this case solution for the scalar field satisfies the Klein - Gordon - Fock equation. We use the selected solution for self-gravitating inflaton field as a background gravitation field in the dynamical chiral field equations. Examples of solutions of field equations for two chiral fields on the various cosmological backgrounds are given in [ $[$ ] $]$.

Consider the equations of the model in a spatially flat Friedmann - Robertson - Walker metric, written in conformal the time $\eta$ :

$$
d s^{2}=a^{2}(\eta)\left(-d \eta^{2}+\gamma_{i j} d x^{i} d x^{j}\right)
$$

where $\gamma_{i j}$ - the metric of the space component of the space-time.

Now, to take in to account the dark sector field influence on the structure formation processes, examine the Einstein and the field equations considering the selected metrics and the basis and chiral space with the assumptions made. In this case the background Einstein equations take the form

$$
\mathscr{H}^{\prime}-\mathscr{H}^{2}=-\kappa K^{(0)}, \quad 3 \mathscr{H}^{2}=\kappa K^{(0)}+a^{2} V(\phi),
$$

where $\mathscr{H}=\frac{a^{\prime}}{a}-$ the Hubble parameter for the conformal time $\eta$, the apostrophe denotes the derivative on the conformal time. The unperturbed equation for the scalar field has the form [W]

$$
\phi^{\prime \prime}+2 \mathscr{H} \phi^{\prime}+a^{2} V_{, \phi}=0 .
$$

Now, in the spatially-flat Universe (D) we can write the dynamical chiral dark sector fields equations with the potential of interaction $W=W(\psi, \chi)$ and the metric coefficient $h_{22}= \pm \psi^{2}$ [Q] (sign «-» in the case of the phantom field $\chi$ ) in the conformal time

$$
\begin{aligned}
\psi^{\prime \prime}+2 \mathscr{H} \psi^{\prime} \mp \psi \chi^{\prime 2}+a^{2} W_{, \psi} & =0, \\
\psi^{2}\left(\chi^{\prime \prime}+2 \mathscr{H} \chi^{\prime}\right)+2 \psi \psi^{\prime} \chi^{\prime}+a^{2} W_{, \chi} & =0 .
\end{aligned}
$$

where $W_{, \varphi^{A}}=\frac{\partial W}{\partial \varphi^{A}}$. The system of equations (可) - (四) is describing the dynamics and the interaction of the dark sector fields on the gravitation field background, which is generated by the inflaton and represented by the solutions of equations (B]) - (田).

As an exact solutions of the Einstain equations (B]) and the iflaton equation (田) we can use solutions, obtained before.

The dark sector fields influence on the long-wave perturbations. We consider an inflationary stage in the evolution of the Universe with dominated the field of the inflation. As already noted, the dark sector fields are comparable in magnitude to the inflaton perturbations. Due to the inequalities on the potentials $W(\psi, \chi) \ll V(\phi)$ and the kinetic terms $K_{12} \ll K^{(0)}$, the perturbed Einstein equations can be written as:

$$
\delta G_{\mu}^{v}=\kappa\left(\delta T_{\mu}^{v}+\Theta_{\mu}^{v}\right) .
$$

Here

$$
\begin{array}{r}
T_{\mu \nu}=\phi_{, \mu} \phi_{, v}-g_{\mu v}\left(\frac{1}{2} \phi_{, \alpha} \phi^{, \alpha}-V(\phi)\right), \\
\Theta_{\mu v}=\psi_{, \mu} \psi_{, v}+h_{22} \chi_{, \mu} \chi_{, v}-g_{\mu v}\left(\frac{1}{2} \psi_{, \alpha} \psi^{, \alpha}+\frac{1}{2} h_{22} \chi_{, \alpha} \chi^{, \alpha}-W_{12}\right) .
\end{array}
$$


We assume that inflation of the Universe is controlled by a scalar field, which has a first-order perturbation $\delta \phi: \phi=\phi_{0}+\delta \phi$. The corresponding perturbation of the stress-energy tensor (8) $\delta T_{\mu}^{\nu}$, according to our model, in order of magnitude satisfies «weak» source with stress-energy tensor of the dark sector fields $\Theta_{\mu}^{v}(\mathbb{Q})$.

We study cosmological perturbations in the longitudinal gauge [ $[1]$, where the perturbed metric has the form:

$$
d s^{2}=a^{2}(\eta)\left\{(1+2 \Phi) d \eta^{2}-(1-2 \Phi) \gamma_{i j} d x^{i} d x^{j}\right\} .
$$

Here $\Phi=\Phi(\eta, \vec{r})$ - the perturbation of the metric (gravitational field). Now from the equation (पD), both parts which is gauge invariant []], we can obtain equations for cosmological perturbations in the longitudinal gauge (10)

$$
\begin{array}{r}
\nabla^{2} \Phi-3 \mathscr{H} \Phi^{\prime}-\left(\mathscr{H}^{\prime}+2 \mathscr{H}^{2}\right) \Phi=\frac{\kappa}{2}\left(\phi_{0}^{\prime} \delta \phi^{\prime}+a^{2} V_{, \phi} \delta \phi+\Theta_{0}^{0}\right), \\
\Phi^{\prime}+\mathscr{H} \Phi=\frac{\kappa}{2} \phi_{0}^{\prime} \delta \phi \\
\Phi^{\prime \prime}+3 \mathscr{H} \Phi^{\prime}+\left(\mathscr{H}^{\prime}+2 \mathscr{H}^{2}\right) \Phi=\frac{\kappa}{2}\left(\phi_{0}^{\prime} \delta \phi^{\prime}-a^{2} V_{, \phi} \delta \phi-\Theta_{*}^{*}\right),
\end{array}
$$

where $\phi_{0}$ - the background solution of the unperturbed equations (B]) and (स) for the inflaton, $\delta \phi=$ $\delta \phi(\eta, \vec{r})$ - the perturbation of the inflaton. The components of the stress-energy tensor of an external source (the spatial components marked with a star) in the conformal time will be: $\Theta_{0}^{0}=$ $\rho=a^{-2} K_{12}+W, \Theta_{*}^{*}=-p=-a^{-2} K_{12}+W$.

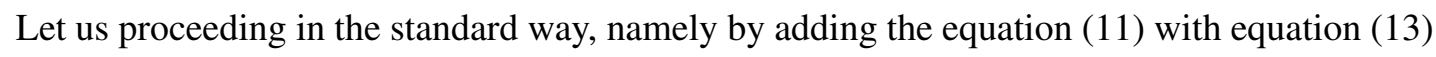
and using the relation to the background equation $\mathscr{H}^{2}-\mathscr{H}^{\prime}=\frac{\kappa}{2} \phi_{0}^{\prime 2}$, then we get the second order differential equation in the partial derivatives with the respect to the variable $\Phi$, which, taking into account $\Theta_{0}^{0}+\Theta_{*}^{*}=\rho-p=2 W$, can be represented as follows:

$$
\Phi^{\prime \prime}-\nabla^{2} \Phi+2 \Phi^{\prime}\left(\mathscr{H}-\frac{\phi_{0}^{\prime \prime}}{\phi_{0}^{\prime}}\right)+2 \Phi\left(\mathscr{H}^{\prime}-\mathscr{H} \frac{\phi_{0}^{\prime \prime}}{\phi_{0}^{\prime}}\right)+\kappa W=0
$$

If we know $\phi_{0}, \mathscr{H}$ from the background equations and $W$, which was introduced into the chiral fields equations (5), ( $(\mathbb{6})$, then we can find the perturbation of the gravitation field $\Phi$ and the corresponding value of the inflaton perturbation $\delta \phi$ from solving the equation (펴), using (미):

$$
\delta \phi=\frac{2}{\kappa \phi_{0}^{\prime}}\left(\Phi^{\prime}+\mathscr{H} \Phi\right) .
$$

The dynamical equation for the inflaton perturbations $\delta \phi$ can be written in the form

$$
\delta \phi^{\prime \prime}+2 \mathscr{H} \delta \phi^{\prime}-\nabla^{2} \delta \phi+a^{2} V_{, \phi \phi} \delta \phi=0 .
$$

We suppose that the universe is in the inflationary stage with the scale factor in the exponential form: $a(t)=a_{s} e^{h_{*} t}\left(a_{s}, h_{*}-\right.$ constants $)$. For the conformal time we have $a(\eta)=-\frac{1}{h_{*} \eta}, \eta=-\frac{e^{-h_{*} t}}{a_{s} h_{*}}$. The solution of the Einstein (B]) and the scalar field (ब) equations in the conformal time, which is corresponding to the exponential expansion of the scale factor, in the cosmological time $t$, will have the form $\mathscr{H}(\eta)=-\frac{1}{\eta}, \phi_{0}=$ const,$V(\phi)=$ const. We note here the restriction on the conformal 
time $\eta: \eta \in\left(-\infty,-1 /\left[a_{s} h_{*}\right]\right)$, which is corresponding to the change of the cosmological time $t$ : $t \in(0, \infty)$.

To solve the dark sector field equations, we must set the type of the chiral space metric components $h_{22}$. Our choice of the chiral space metric components $h_{22}= \pm \psi^{2}$ [Q] is tentative, and, as a rule, allows make a calculation of results in more complicated cases.

Equations $(\mathbb{W})-(\mathbb{1} 3)$ given for inflationary stage this result:

$$
\Phi^{\prime \prime}-\nabla^{2} \Phi+\kappa W(\eta)=0
$$

Using the standard way [ए]] we find the solutions of (피) in the form:

$$
\Phi(\eta, \mathbf{x})=\Phi(\eta) e^{i \mathbf{k} \mathbf{x}}
$$

The term $\nabla^{2} \Phi$ can be neglected in the long-wave approximation at the low wave number $k$. The solution of the equation in this case is the time-dependent function $\Phi(\eta)$. We find the corresponding

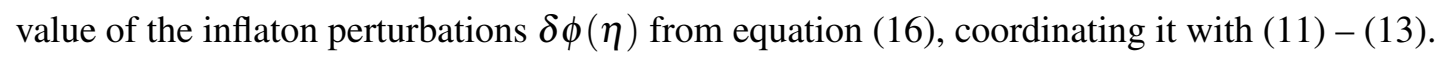

If a «weak» source is absent $\left(\Theta_{\mu}^{v}=0\right)$, that is, in the usual case of the inflationary perturbations, we have the solution, coincides with that given in [ए]]:

$$
\Phi^{(0)}=C_{2} \eta, \quad \delta \phi^{(0)}=C_{1} \eta^{3}+C_{3} .
$$

Direct calculations using the dynamic equation for the inflaton perturbations (며) shows the equality of the perturbation $\delta \phi=\delta \phi^{(0)}$ independent from the parameters of a weak source with the stress-energy tensor (Q9).

We assume that the potential $W$ depends only on $\psi$ and consider the potential of the Higgs type

$$
W(\psi)= \pm \frac{h_{*}^{2} \gamma^{2}}{4 \beta^{2}} \psi^{4}+h_{*}^{2} \psi^{2}+W_{*}
$$

In this case, the solution for the dark sector fields are proportional to the conformal time $\eta: \psi=\beta \eta$,

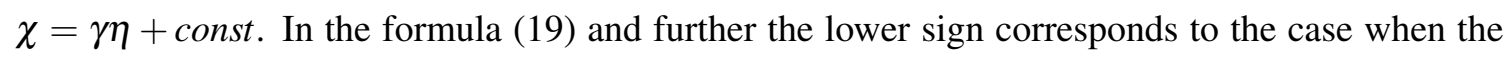
field $\chi$-phantom. The potential and the kinetic energy of the conformal time dependence is given by:

$$
\begin{array}{r}
W(\eta)=h_{*}^{2} \beta^{2} \eta^{2}\left(1 \pm \frac{\gamma^{2}}{4} \eta^{2}\right)+W_{*}, \\
K(\eta)=\frac{1}{2} h_{*}^{2} \beta^{2} \eta^{2}\left(1 \pm \gamma^{2} \eta^{2}\right)
\end{array}
$$

here $W_{*}, \beta, \gamma$ - the integration constants. Next, we substitute $W(\eta)$ in the (ㅍ).

$$
\Phi_{1}=C_{2} \eta \mp \frac{\kappa\left(h_{*} \beta \gamma\right)^{2}}{120} \eta^{6}-\frac{\kappa\left(h_{*} \beta\right)^{2}}{12} \eta^{4}-\frac{\kappa W_{*}}{2} \eta^{2} .
$$

Note that this solution will be agreed with the equation (피) if we require $W_{*}=0, \beta \ll 1$ and $\beta \rightarrow 0$ when $\eta \simeq \eta_{\text {infl }}$. Another possibility, which we do not consider here, is associated with the presence of a non-zero component of $\Theta_{0}^{*}$ (see [焑]). 
Let us consider the physical properties of the solution ([22). For this we find the equation of state of dark sector fields

$$
w=\frac{K_{12}-W}{K_{12}+W}=\frac{p_{\sigma}}{\rho_{\sigma}}
$$

and present a graphical illustration of the solution ([20) - (223). The corresponding dependence of the potential $W$, the kinetic energy $K_{12}$ and the equation of state parameter $\omega$ on the time for the case when both fields are not phantom is shown in Fig. $\mathbb{W}$. The graph shows the potential of a solid line, the kinetic energy - dotted, the equation of state parameter - the dot-dashed.

Recall that the equation of state parameter for dark energy, which provides the accelerated expansion of the universe satisfies the inequality: $w<-1 / 3$ [3]. Time of inflation in the selected time scale of cosmic time ends at the point $t=2$ (see $\mathbb{W}$ ), because after this point the equation of state parameter $w$ is almost constant and corresponds to dark energy. Note that the behavior of functions in a negative time is presented for completeness of their evolutionary development.

The gravitation field perturbations $\Phi_{1}$ (2) shown on Fig. [ $\square$ of a dotted line (for the phantom case - the dot-dash), only the inflaton perturbation $\Phi^{(0)}(\mathbb{1 1 8})$ - the solid line to compare. From figure $\square$ we can see, that the «weak» source in the form of the dark sector fields weakens the inflaton perturbations of the gravitational potential $\Phi^{(0)}$ (피).

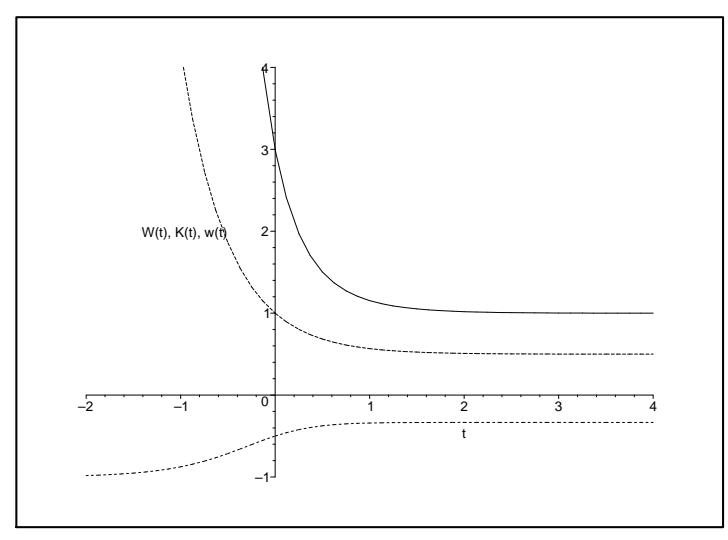

Figure 1: The parameters of the dark sector fields, describing by the NSM

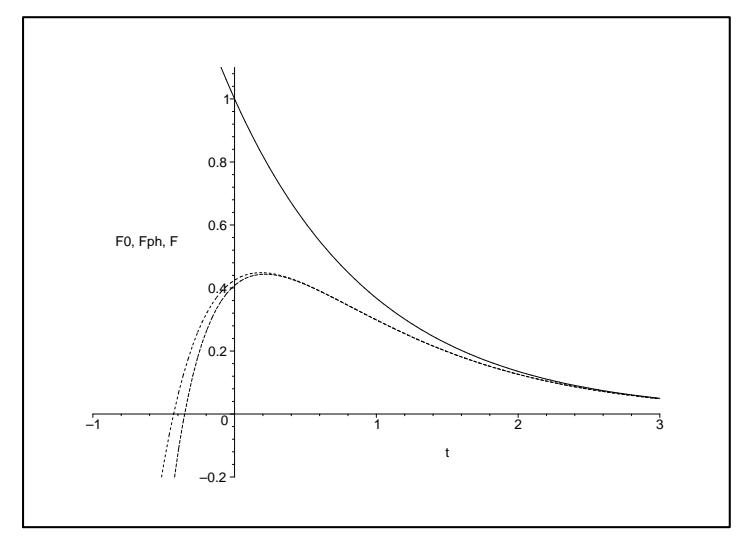

Figure 2: The perturbations of the gravitational potential

The above solution demonstrates a common pattern for the other cases, depending on the parameters of the «weak» source on time, including the case of the phantom field [Q]. The resulting gravitational potential $\Phi$ has approximately the same shape and is slightly different for small time for the different background solutions for chiral dark sector fields.

Conclusions. Early cosmological inflation as a process of super-fast expansion of the universe from the physical point of view can regarded as an expression of the strong gravitational field, generated by the self-interacting scalar field. In this case, the link «gravitational field - inflaton» is a self-gravitating «stiff» physical system, which is primary in relation to other possible material components present at a very early stage of evolution of the universe. For this material components, we can assume the existence of more «weak» dark sector fields, such as: the axion, the quintessence and the phantom fields. 
In our approach, we assume that at the stage of early inflation the cosmological dark sector fields have an impact on the level of the cosmological perturbations of the gravitational field and the inflaton, thus affecting the formation of large-scale structure. The obtained solution confirms the change of the perturbation of the gravitational field for the long-wave approximation, which describes the formation of large-scale structure of the universe. Analysis of other solutions [ $Q$ ] confirms that the presence of a «weak» source reduces the gravitational field perturbation in the long-wave approximation.

This work realized with partial financial support for program of the Russian-Indian cooperation RFBR (Grant 08-02-91307- IND_a) and DST (Grant RUS P/84 - DST). The work of O. G. Panina is also supported by RFBR (Grant 11-02-16115-mob_z_ros). O. G. Panina is grateful to the Scientific Research Laboratory of Mathematical Physics for useful discussions.

\section{References}

[1] V. Sahni, A. Starobinsky. The Case for a Positive Cosmological Lambda-term. Int. J. Mod. Phys. D 9, 373 (2000).

[2] V. Sahni, A. Starobinsky. Reconstracting Dark Energy. Int. J. Mod. Phys. D 15, 2105 (2006).

[3] E. J. Copeland, M. Sami, S. Tsujikawa. Dynamics of Dark Energy. Int. J. Mod. Phys. D 15, 1753 (2006).

[4] S. Tsujikawa. Dark Energy: investigations and modelling, ArXiv:1004.1493 (2010).

[5] S. V.Chervon. Izv.Vyssh.Ucheb.Zaved. Fiz. 5, 114 (1995).

[6] S. Veeraraghavan, A. Stebbins. Causal Compensated Perturbations in Cosmology. Astrophys. J., 365, 37 (1990).

[7] A. H. Jaffe. Quasi-Linear Evolution of compensated cosmological perturbations. Phys. Rev. D, 49, 3893 (1994).

[8] O. G. Panina, S. V. Chervon. Electronic journal «Investigated in Russia», 093/091021, 1190 (2009). http://zhurnal.ape.relarn.ru/articles/2009/093.pdf

[9] S. V. Chervon, O. G. Panina. Cosmological perturbations and dark sector fields. Journal «Vestnik UISPU: sbornik nauchnih statej». Ulyanovsk: UlSPU. Vol.6, 196 (2010).

[10] S. V. Chervon. Nonlinear fields in gravitation theory and cosmology.- Ulyanovsk: UlSU, (1997).$191 \mathrm{p}$.

[11] V. F. Mukhanov, H. A. Feldman, R. H. Brandenberger. Theory of Cosmological Perturbations. Phys.Rep., 215, 203 (1992).

[12] D. Polarski, A. A. Starobinsky. Isocurvature Perturbations in Multiple Inflationary Models. Phys.Rev. D 50, 6123 (1994). 